\title{
Evidenzbasierte Therapie in der gastroenterologischen Onkologie
}

Bislang galt für maligne gastroenterologische Neoplasien folgendes Diktum: Nur die operative Resektion bietet die Chance einer definitiven Heilung. Chemotherapie oder Bestrahlung haben in der Regel nur einen Stellenwert in der palliativen Therapie. Auch endoskopische Maßnahmen beschränken sich auf die Palliation, z.B. bei der Überbrückung einer malignen Gallenwegstenose mittels Stent. Die Therapie von malignen und prämalignen Neoplasien muss heute aber differenzierter gesehen werden. $\mathrm{Zu}$ den therapeutischen Standardverfahren hinzugetreten sind endoskopische Verfahren wie die Mukosaresektion oder die photodynamische Lasertherapie. Auch die chemotherapeutischen Möglichkeiten unterliegen einem Wandel. 5-Fluorouracil ist zwar das «Standard»-Chemotherapeutikum bei vielen gastroenterologischen Neoplasien geblieben. Neue Substanzen, wie Gemcitabin, Capezitabin, Irinotecan, Oxaliplatin, Gefitinib, haben aber je nach Tumorart und Stadium ihren Stellenwert, wie durch kontrollierte Studien belegt wurde.

Um der Komplexizität in der Diagnostik und Therapie gastroenterologischer Neoplasien gerecht zu werden, ist die interdisziplinäre Zusammenarbeit in «Tumor Boards» unabdingbare Voraussetzung. Chirurg, Gastroenterologe/Onkologe, Strahlentherapeut, Radiologe, Nuklearmediziner und Pathologe legen gemeinsam das diagnostische und therapeutische Prozedere fest. Je nach Tumorart, Lokalisation und Stadium werden die kurativen, neoadjuvanten, adjuvanten und palliativen Optionen diskutiert. Für die Entscheidungsfindung ist die Kenntnis der wissenschaftlichen Studienlage Voraussetzung. Wenn für eine therapeutische Entscheidung Belege durch Studien fehlen, sollte immer besprochen werden, ob der Patient nicht in eine laufende Studie zu dieser Frage eingeschlossen werden könnte.

Das vorliegende Schwerpunktheft versucht am Beispiel dreier häufiger gastroenterologischer Neoplasien, dem Ösophagus-, dem Magen- und dem kolorektalen Karzinom, das derzeitige «evidenzbasierte» Vorgehen aufzuzeigen. 